\title{
Energy Efficient Architectures and Techniques for Green Radio Access Networks
}

\author{
(Invited Paper) \\ Jianhua He, Pavel Loskot, Tim O'Farrell \\ School of Engineering \\ Swansea University \\ Swansea, UK \\ \{j.he, p.loskot, t.ofarrell\}@swansea.ac.uk \\ Simon Armour \\ Centre for Communications Research \\ Bristol University \\ Bristol, UK \\ Simon.Armour@bristol.ac.uk \\ Vasilis Friderikos \\ Centre for Telecommunications Research \\ Kings College London \\ London, UK \\ vasilis.friderikos@kcl.ac.uk \\ John Thompson \\ School of Engineering \\ University of Edinburgh \\ Edinburgh, UK \\ John.Thompson@ed.ac.uk
}

\begin{abstract}
Energy consumption in wireless networks, and in particular in cellular mobile networks, is now of major concern in respect of their potential adverse impact upon the environment and their escalating operating energy costs. The recent phenomenal growth of data services in cellular mobile networks has exacerbated the energy consumption issue and is forcing researchers to address how to design future wireless networks that take into account energy consumption constraints. One fundamental approach to reduce energy consumption of wireless networks is to adopt new radio access architectures and radio techniques. The Mobile VCE (MVCE) Green Radio project, established in 2009, is considering such new architectural and technical approaches. This paper reports highlights the key research issues pursued in the MVCE Green Radio project.
\end{abstract}

\section{INTRODUCTION}

Climate change and the need to reduce energy consumption is an imperative impacting all of our society, and this is also the case for mobile and wireless networks. For example, considering a 24 hour UK digital broadcast TV network, its average power consumption is about $2 \mathrm{MW}$. In comparison, a typical mobile phone network in the UK will consume approximately $40 \mathrm{MW}$, excluding the power consumed by the users' handsets, which is a significant proportion of the total energy consumed by the information technology industry. Further, today's always-on IP data services are significantly increasing the energy consumption of cellular mobile networks without increasing revenues. From the operator and manufacturer perspectives, there are increasing energy costs with larger base station site densities and rising energy price trends. In addition, operators and manufacturers have pledged to reduce carbon emissions and the environmental impact of wireless networks. For example, Vodafone has set a group target to reduce its $\mathrm{CO}_{2}$ emissions by $50 \%$ by 2020 , from $2006 / 07$ levels [1]; while Orange has set a target to reduce its greenhouse gas emissions per customer by $20 \%$ between 2006 and 2020 [2]. Thus, carbon footprint and energy operating costs have become vital constraints in future system design.

Analysis of a typical cellular mobile network readily reveals that the most energy is consumed in the radio access network (RAN) as illustrated in Figure 1 [3]. Accordingly, the MVCE Green Radio project is focused on developing new architectures and radio techniques to achieve a desired 100 -fold reduction in the energy consumption of the RAN compared with 2009 levels. However, achieving this energy reduction must be done without compromising the Quality of Services (QoS) experienced by the end-users and without displacing the energy consumption to other parts of the overall network. For these reasons, the Green Radio project considers not only the RF energy consumed for communications but also operational, embodied and backhaul energy consumption routes.

The MVCE Green Radio project is pursuing energy reductions within two major complementary research streams as follows:

- Architectural aspects of the Green Radio explores new RAN approaches that significantly reduce energy consumption;

- Radio techniques for power reduction in the Green Radio explore novel approaches that can be used in the base stations and the handsets to reduce energy consumption in the network.

The desire to achieve a 100 -fold reduction in energy consumption is being addressed through focused studies on novel architectures and radio techniques. This paper provides an overview of these approaches and reports the key findings and principles that are emerging from the research program going forward.

The paper is structured as follows. Section 2 provides a discussion of the energy metrics used to quantify the RAN energy consumption. Section 3 provides an overview of the architectures and radio techniques that are studied in order to improve the energy efficiency. Section 4 details research case studies on RAN architectures and techniques. The conclusions are then drawn in Section 5. 


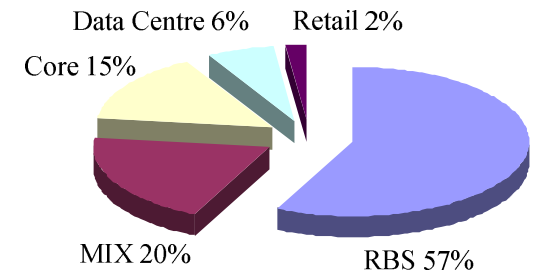

Network

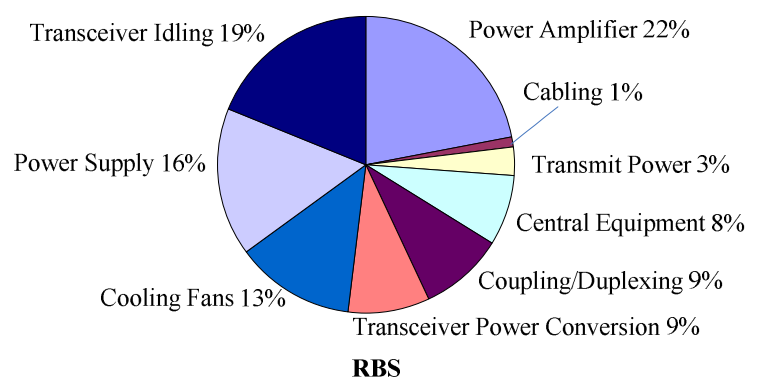

Figure 1: Contributions to electricity use in mobile phone networks and radio base stations (RBS) [3].

\section{ENERGY METRICS AND BUDGETS FOR RADIO ACCESS NETWORKS}

Numerous energy metrics have been defined in order to quantify the energy consumption of networking equipment. Many of these definitions can be found in the open literature, and more recently, the energy metrics has been standardized by several institutions. It is expected that the new standardized energy metrics and the corresponding standardized measurements of the power and energy consumption will emerge in the near future. In general, the energy metrics are defined as the energy consumption normalized per some quantity or network entity. For instance, the energy consumption is normalized by the peak data rate, the number of subscribers, the communication distance and the coverage or service area. The purpose of the energy metrics standardization efforts so far is to achieve the energy consumption compliance of the network equipment. On the other hand, the energy metrics in the open research literature are usually considered more broadly taking into account the specific system models considered. The standardized energy metrics are usually variations of the following two basic definitions: the energy consumption ratio (ECR) [4] and the telecommunications energy efficiency ratio (TEER) [5]. The ECR metric is defined as the ratio of the peak power (in Watts) to the peak data throughput, i.e.,

$$
\mathrm{ECR}=\frac{\text { power }}{\text { rate }}\left[\frac{\mathrm{Watt}}{\mathrm{bps}}=\frac{\text { Joule }}{\text { bit }}\right]
$$

The TEER metric is more general than the ECR metric and it can be written as a ratio of TEER= useful work/power. The units of the TEER metric depends on the specific quantity considered to be the useful work. It is also possible to express the powers in $\mathrm{dB}$ or $\mathrm{dBm}$ units and modify the ECR and the TEER metrics accordingly although this possibility is not currently considered in the standards. Also, the standards usually do not explicitly specify the definitions of the power used in the ECR and TEER metrics, e.g., whether or not the power considered accounts for the RF power only.

In the GR project, the ECR metric is adopted by assuming the average powers and the average data rates rather than their peak values, i.e.,

$$
\mathrm{ECR}=\frac{E}{M}\left[\frac{\text { Joule }}{\text { bit }}\right]
$$

where $E$ is energy required for delivering $M$ bits to the application layer. The ECR metric is evaluated either for the whole RAN as well as for one cell or cell sector of the RAN assuming a given RF average transmission power and a given average throughput in each cell. Furthermore, the energy consumption gain (ECG) metric has been defined in the GR project as a ratio of the ECR metrics of the two systems under consideration, for example, a baseline reference system and a system with a more energy efficient RAN architecture. Consequently, the ECG metric quantifies the energy consumption improvement relative to the common reference system. In some scenarios, the energy reduction gain (ERG) (expressed in percent) is preferable. The ERG metric is derived from the ECG metric as

$$
\mathrm{ERG}=\left(1-\frac{1}{\mathrm{ECG}}\right) \times 100[\%]
$$

\section{ENERGY EFFICIENT RAN ARCHITECTURES AND TECHNIQUES}

This section describes the RAN architectures and radio techniques being studied in the Green Radio project. The architectures and radio techniques are divided into several research themes as explained below. While research is focused on the LTE, the LTE-Advanced technologies, and the HSPA the systems beyond $4 \mathrm{G}$ are also considered.

\subsection{Base Station Design}

The overall efficiency of the base station is governed by the power consumption of its various constituent parts and the core radio devices: radio transceivers, power amplifiers and transmit antennas. Base stations also contain other auxiliary equipment providing facilities such as connection to the service provider's network and the climate control. A major opportunity to achieve the power reduction lies in developing techniques to improve the efficiency of base station hardware.

Research work in Green Radio project obtained models for various base station configurations (macrocell, microcell, picocell and femtocell) in order to establish how improvements in the hardware components will impact the overall base station efficiency. The starting point for this analysis has been the transmit chain. Near-market power consumption figures have been used in order to establish a benchmark efficiency figures against which improvements made as part of the project can be assessed. Target power consumption figures allow future overall base station efficiencies to be predicted.

\subsection{Cell Deployment Topologies}

This research seeks to characterize two competing premises. For large-cell deployments, one type of BS equipment is 
commonly used for a range of cell-sizes. For example in LTE, using the same type of Remote Radio Unit (RRU) for cells of diameters from $2000 \mathrm{~m}$ to $500 \mathrm{~m}$ will result in higher than necessary energy consumption in the smaller cells. In this case, energy reduction is sought, for example, by using antenna technologies that can support multiple, steerable antenna beam patterns to cover a geographic area with the least number of cell sites. In contrast, for small cell deployments the RF power required to achieve a target throughput/coverage level reduces substantially with decreasing cell size. If the overall BS power consumption can be scaled with the RF transmitted power, then substantial energy savings across the RAN are possible. However, as the total number of BS is increased in order to cover a given geographical area, any reduction in RF transmitted power is counteracted by inefficiency, embodiment and backhaul energy overheads accrued by cell replication. Thus research into small cell deployments is focused on enabling approaches to scale energy consumption with teletraffic load by only fully powering infrastructure when and where it is needed.

\subsection{Femtocell Deployments}

One of the aims of this research is to investigate the energy consumption characteristics of femtocell deployment in a macrocellular network from a system-wide perspective. Femtocells provide excellent means for studying small cell scenarios based on their swift and practical integration into the existing cellular network. The scenario of an urban hierarchical cellular network is being considered, with open access femtocells deployed by subscribers in their households. The total system energy consumption includes the equipment's embodied as well as operational energy. More specifically, the network equipment considered for energy consumption calculation includes macrocell BSs, femtocell access points and mobile terminals. For an efficiency comparison between different scenarios, the system energy consumption is divided by the number of the users in the network. QoS is taken into account by including the average transmission bandwidth as a QoS measure. Simulations are implemented based on different macrocell and femtocell capacities. The Indifference Curves technique is used to evaluate the energy consumption and QoS provisioning levels, by adjusting the percentage of femtocell deployed or used.

\subsection{Multihop Architectures and Routing}

The use of relays to exchange information between a base station and mobile terminal is an efficient way to improve base station energy efficiency. This is because the transmission distance can be reduced, increasing data rates or permitting reductions in transmission energy. Relays can enable important reductions of the network energy consumption without complicated infrastructure modifications. These may be deployed in streets or in buildings to provide improved signal quality to locations which might otherwise experience poor QoS. One important research objective in this area is to compare the energy efficiency of relay techniques with the use of femtocells.

Store carry and forward (SCF) relaying provides a generalization of previous relaying techniques that have to transmit an information message as soon as received and in the most general application SCF allows mobile users to connect to access points and/or wireless networks in which they do not have immediate connectivity, i.e., can not be reached via single hop transmission. More specifically, SCF relaying allows delay tolerant messages to be transmitted via multi hop relaying to nodes which can potentially store the message and forward it to suitable access points based on various optimization criteria [6]. Being able to control and postpone transmission of elastic traffic in future time intervals using SCF relaying can have a number of significant benefits in future wireless networks. The SCF networking paradigm has the potential to significantly curb energy consumption level and also to allow the decongestion of hot-spot areas by migrating traffic to neighbour cells or even shutting down cells with very low utilization by transferring their limited traffic to cells in the close vicinity. Another auxiliary benefit of implementing SCF relaying is the inter-cell interference can be dramatically reduced since transmission are localized which can lead to better spatial utilization of scarce wireless resources.

\subsection{Advanced Spectrum Management}

Recent developments, both from regulatory and technical perspectives, are allowing freer use of spectrum [9][10]. Moreover, in future contexts, operators can expect to have access to an increasingly wide range of spectrum bands, of vastly differing physical characteristics. In this scenario, operators will be able to use their spectrum bands for whichever technologies they see fit, albeit subject to constraints such as transmission power mask and perhaps implied interference. In Green Radio, advanced spectrum management techniques involving dynamic spectrum access of the range of spectrum bands available to the operator are being explored as means of reducing RAN energy consumption. Investigations are focused on four key areas: 1) dynamically moving users from other bands into particularly active spectrum bands, allowing radio network equipment in those other bands to be partially or fully powered down; 2) dynamic spectrum access in order to take advantage of better propagation spectrum and reduce necessary transmission power; 3) dynamic spectrum access to allow channel bandwidths to be increased hence transmission powers to be decreased; and 4) the better hierarchical management of spectrum where different cell types coexist.

\subsection{Resource Allocation Strategies}

We use the term resource allocation to describe how the base station transmitter make the decision of how and when to transmit data to different users on the downlink (basemobile link) within the cell it is serving. Resource allocation techniques that make the most efficient use of the RF amplifier have the potential to improve energy efficiency significantly. Such energy reductions could lead to further energy savings through switching off transceiver equipment and base station cooling. In addition, analysis of data traffic in wireless networks show that the traffic load is typically very uneven across the cells. In an analysis of 200 cells in [2, Chapter 9], it is shown that even in peak hours, $90 \%$ of the data traffic is carried by only $40 \%$ of the cells in the network. 
Therefore, techniques that minimize energy consumption across varying traffic load conditions are an important research direction. Two complementary techniques are being studied within the MVCE GR project aimed towards low and high traffic load conditions, respectively. Under low traffic load conditions a frequency domain approach is used which exploits spare bandwidth to reduce energy consumption. When the traffic load is high, an opportunistic resource scheduling and allocation strategy is investigated to exploit multiuser diversity to increase the overall capacity and reduce energy consumption.

\subsection{Interference Management and Mitigation}

Interference cancellation schemes are indispensable to combat interference in any practical communication systems where multiple base stations share the same spectrum. The impact of interference is more severe as the users move closer to the cell edge, leading to significant data rate reductions in such regions. Most existing interference cancellation schemes have been designed to increase the spectral efficiency and data rate, while overlooking the energy efficiency. However, research efforts in the Green Radio program are focused on developing energy efficient interference cancellation schemes. There are two complementary strategies, namely distributed antenna systems and receiver interference cancellation.

\section{CASE STUdies}

In this section several research case studies are presented which report up-to-date finding and trends.

\subsection{Cell Deployment Strategies for Wireless Wide Area Networks}

This research considers the impact of the cell size and antenna downtilt on the RF energy consumption of an LTE RAN [1] while accounting for intercell interference. A homogeneous deployment of cells for a fixed defined geographic area and user density are assumed. The objective is to determine the cell size and the BS antenna tilt that give the most energy savings without violating a predefined operating QoS constraint for LTE. A joint QoS constraint is imposed based on the combined requirement to meet a given minimum cell throughput-coverage target and a given cell average throughput. The cell throughput-coverage target was set to $1 \mathrm{Mbit} / \mathrm{s}$ over $95 \%$ of the cell area while the cell average throughput target was set to $4.5 \mathrm{Mbit} / \mathrm{s}$. The least transmit power to meet the cell throughput-coverage target was found for a given cell diameter and BS antenna height while varying the BS antenna downtilt. The results shown in Figure 2(a) demonstrate that ECR decreases monotonically with cell diameter and downtilt angle under a throughputcoverage constraint. Figure 2(b) shows that while all the downtilts fulfill the minimum cell throughput-coverage target, only downtilts $6^{\circ}$ and $5^{\circ}$ fulfill the average throughput target. Figure 2(b) also indicates that larger cells meet the joint QoS constraint more tightly than smaller cells. That is, in small cells the ECR is constrained by the throughputcoverage target well before the average throughput target.
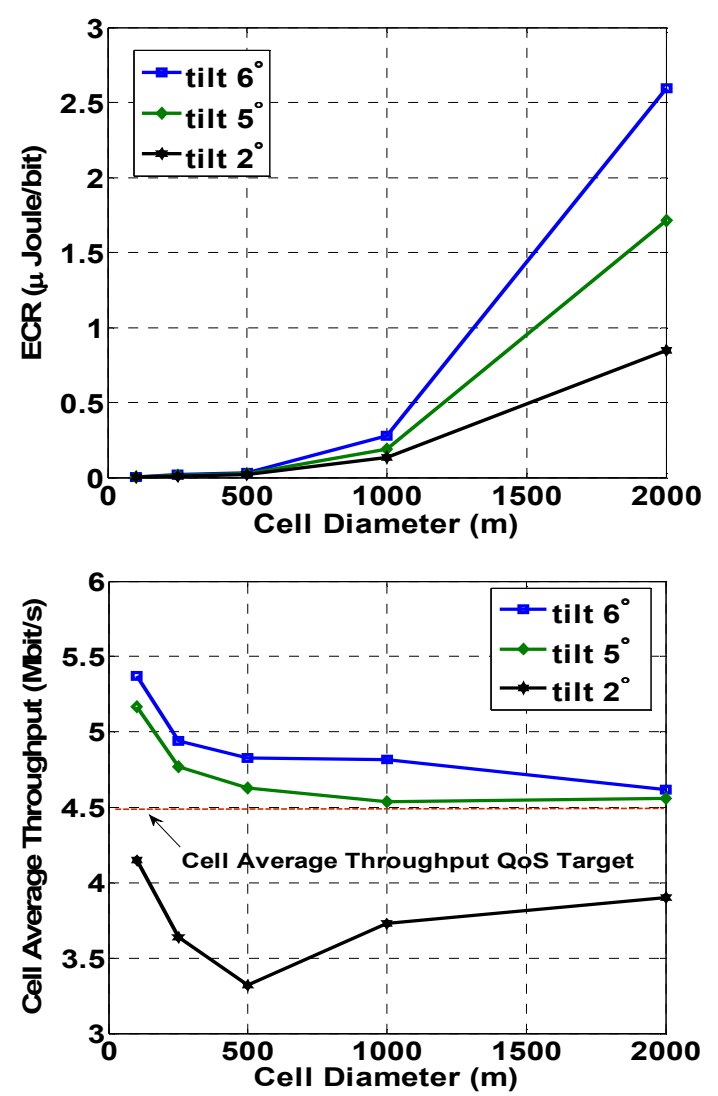

Figure 2: (a) ECR and (b) Average Cell Throughput versus cell diameter

\subsection{Multihop Networking}

As a road map for future research on SCF relaying there are several challenges that need to be tackled. An important issue is to provide an overhead characterization for establishing the overlay vehicular network in terms of communication cost and computational complexity. In addition, validation and quantification of the benefits on real data and under various environments should be performed. Finally, further research need to address greedy low complexity and decentralized algorithms for SCF relaying in cellular networks. In this case study mobile relay nodes are considered in the multihop cellular network under investigation. Unlike conventional forwarding mechanisms, the SCF technique is realized within the cell to physically propagate messages. In SCF informed routing decisions are made both in space and time. In this investigation a timeexpanded network has been derived and a general optimization problem has been formulated and shown to have linear complexity over the network size. Here, a single cell scenario with 20 uniformly distributed active source nodes and 40 candidate mobile relays are considered. Each source node has a file of 4 Mbits to send to the base station. The proposed SCF scheme is compared to a basic multihop $(\mathrm{BMH})$ relaying strategy where messages are forwarded as soon as they become available. The ECR results of these two schemes are presented against the tolerable message delivery delay in Figure 3. The results in Figure 3 show that a reduction in ECR can be obtained by trading off message delivery delay. Larger energy consumption reductions can be obtained for increased cell sizes with higher end-to-end 
message delivery delays. Compared to the BMH relaying strategy, the SCF scheme shows an enhanced energy reduction performance for elastic message deliver delay constraints. The enhanced performances of the SCF scheme can be explained by the longer period that relay nodes carry information. However, with SCF relaying improvements in energy savings are possible for the same limiting delay achieved by the BMH scheme. Indicatively, for a tolerable delay of 14 seconds, the SCF scheme achieves more than $40 \%$ reductions in the communication energy consumption compared to the $\mathrm{BMH}$ paradigm.

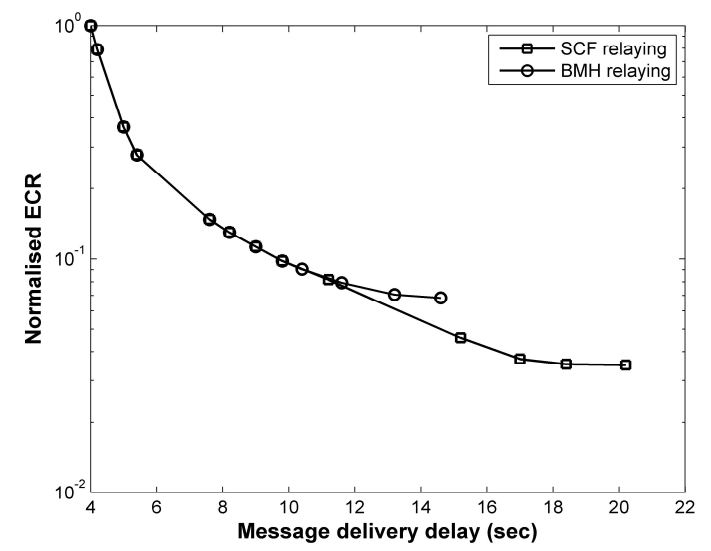

Figure 3: Performance of SCF and BMH relaying in terms of ECR versus message delivery delay.

\subsection{Resource Allocation Strategies}

Under low traffic load conditions, the base station is likely to have more bandwidth available to transmit data to users than is actually required at that time. Hence, it is possible to exploit trade off between spectral and energy efficiency to achieve the energy savings while retaining the quality of service [5]. Figure 4(a) shows predicted ECG gains as a function for the signal-to-interference and noise ratio (SINR) required at the mobile receiver for a given data rate. The value of $\alpha$ specifies the permitted bandwidth expansion and curves are shown for values of $\alpha$ in the range 2-6. The results show that as the SINR increases, so does the potential improvement in ECG from using the bandwidth expansion technique. When the traffic load is high, the base station may be transmitting data to many users simultaneously, possibly using MIMO techniques. In this case, it is usually possible to exploit multiuser diversity to increase the overall multi-user capacity achieved via an opportunistic resource scheduling and allocation strategy. A link adaptation approach can be taken into consideration to ensure the most energy saving transmission mode is employed within the allocated resource for a required QoS level. As an example from [6], Figure 4(b) shows the performance of different MIMO precoding schemes measured using a single-user MIMO diversity scheme space frequency block coding (SFBC). The schemes using higher degrees of diversity achieve a lower cost in terms of energy for each information bit.
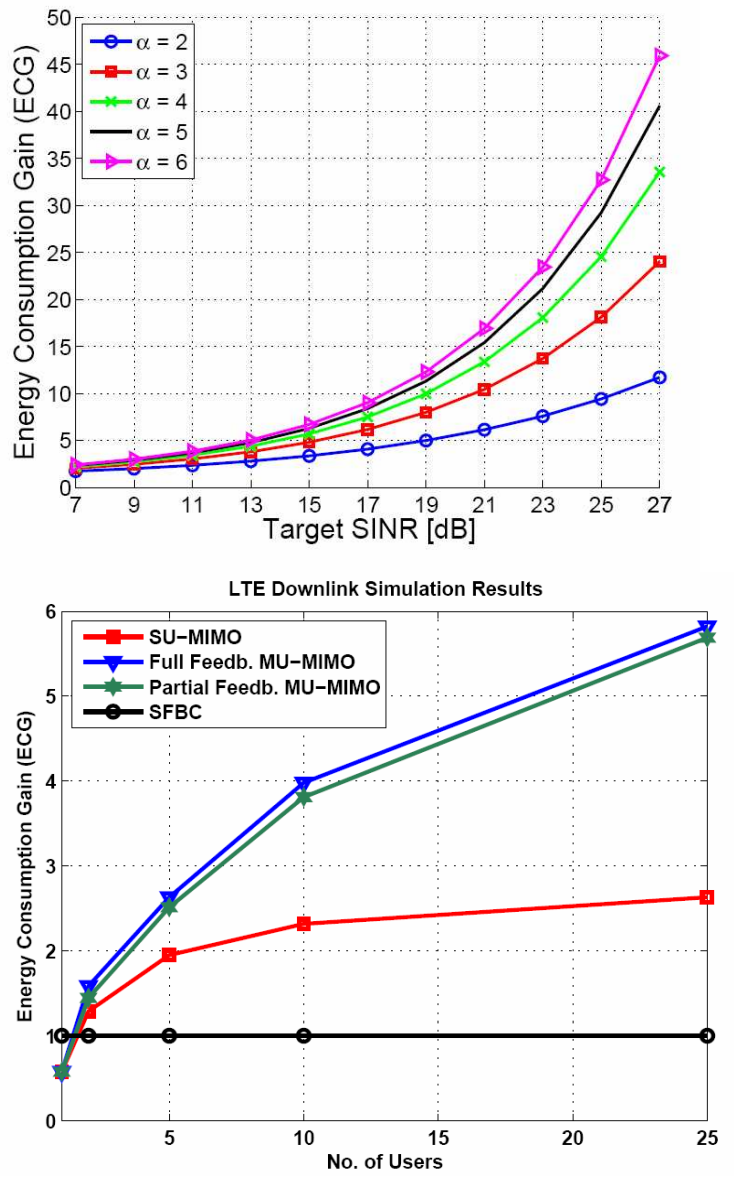

Figure 4: (a) ECG of frequency domain bandwidth expansion as a function of required SNR; (b) ECG of various MIMO schemes, relative to $\mathrm{SFBC}$ all at $3 \mathrm{bits} / \mathrm{s} / \mathrm{Hz}$ spectral efficiency.

\subsection{Interference Management and Mitigation}

One way to reduce interference in cellular systems is to coordinate the multiple antennas of the adjacent base stations to form a Distributed Antenna System (DAS) [7]. For the resulting coordinating DAS, each and every cell edge user is collaboratively served by its surrounding base stations rather than only by the single best base station. This permits the interference to users on the cell edge to be effectively controlled and mitigated by coordinated beamforming among the DAS antennas. The following three schemes can be used by coordinating downlink beamforming: (1) The user is served by only one base station while other base stations avoid transmitting signal energy towards that user; (2) All users are served by multiple base stations using multiple antenna beam-forming and coherent user-end combining, i.e., exploitation of the degrees of freedom offered by the DAS; (3) Users are allocated to one or more base stations based on their position. These three schemes are compared in terms of ECG vs SINR against the conventional non-cooperative case, in Figure 5 for a cluster of 3 cells with one user per cell. The results show that all three schemes significantly outperform the conventional system at high SINRs, with schemes 2 and 3 outperforming scheme 1 . However, scheme 1 may be preferable over schemes $2 / 3$ in practical implementation, since it requires much less signalling data to be exchanged 
between the base stations and hence less energy consumption.

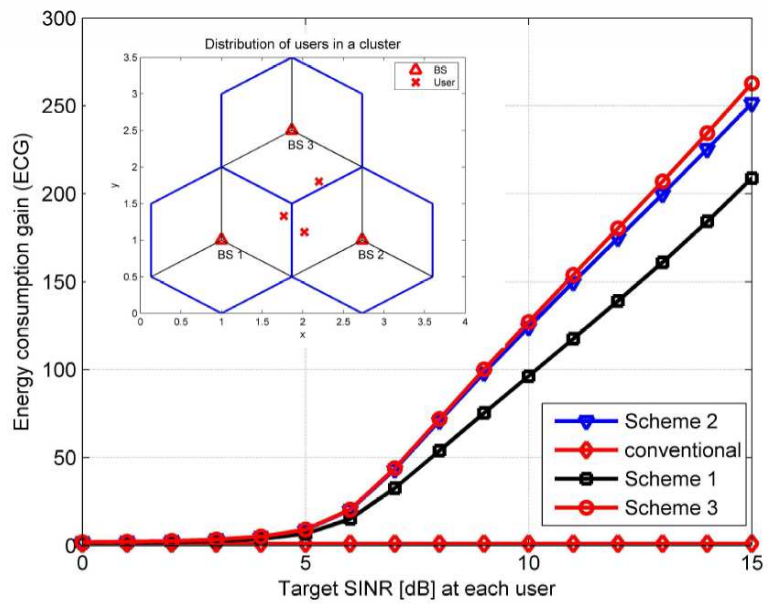

Figure 5: Performance comparison of ECG (relative to no DAS case) vs SNR for 3 DAS schemes.

\section{CONCLUSION}

Energy consumption aspects of the cellular RANs have been investigated, and the recent developments in the emerging research area of the energy-efficient RANs have been outlined. The RANs are responsible for the largest proportion of total energy consumption in a cellular mobile network, with the RBSs accounting for more than half of the energy consumption of the RAN. The main focus of this paper has therefore been on the possible energy reductions in the RANs by optimizing existing network architectures, and by introducing novel underlying transmission concepts and radio techniques. The energy saving strategies presented in this paper are some of the promising approaches that are currently being investigated within the MVCE Green Radio project, aiming at achieving a desired 100 -fold reduction in the energy consumption of cellular networks. Specifically, the following strategies to improve energy efficiency of present and future networks have been studied: base station design, cell deployment topologies, multihop relaying, spectrum management, radio resource allocation, interference management and mitigation, and energy efficient routing. The details of this research are available to the MVCE researches in technical research reports.

\section{ACKNOWLEDGEMENTS}

The work reported has formed part of the Green Radio Core 5 Research Programme of the Virtual Centre of Excellence in Mobile \& Personal Communications, www.mobilevce.com. This research is funded by the Industrial Companies who are members of Mobile VCE and by the UK EPSRC under Grant Numbers EP/G060584/1, EP/G06041X/1, EP/G062420/1 and EP/G064105/1.

The authors express their grateful thanks to the following contributing researchers: H. Aghvami, B. Badic, P. M.

Grant, H. Haas, H. Hamdoun, C. Han, L. Hanzo, T. Harrold, O. Holland, Y. Hou, P. Kolios, I. Krikidis, I. Ku, D.
Laurenson, T. A. Le, M. R. Nakhai, D. Quintas, S. Videv, C.-X. Wang, J. Zhang

\section{REFERENCE}

[1] http://www.orange.com/en_EN/tools/boxes/documents/att00005072 CSR_report 2007.pdf

[2] http://www.vodafone.com/etc/medialib/attachments/cr_downloads.

[3] H. Karl, "An overview of energy-efficiency techniques for mobile communication systems," Telecommunication Networks Group, Technical University Berlin, Tech. Rep., September 2003.

[4] Exploratory group on green (EGG) report and recommendations, Alliance for Telecommunication Industry Solutions (ATIS), Washington, DC (USA), Report Draft 5.0, 2009.

[5] "Network and telecom equipment - energy and performance assessment test procedure and measurement methodology," ECR initiative, Deutsche Telekom AG, Germany, Tech. Rep., 2008.

[6] K. Fall and S. Farrell, "DTN: An Architectural Retrospective," IEEE Journal on Selected Areas in Communications, Vol. 26, No. 5, Jun. 2008, Page(s):828 - 836.

[7] http://www.irtf.org/charter?gtype=rg\&group=dtnrg

[8] V. Chandrasekhar, JG Andrews, A. Gatherer, "Femtocell networks: a survey," IEEE Communications Magazine, 2008.

[9] Ofcom, "Spectrum Usage Rights: Technology and Usage Neutral Access to the Spectrum" Consultation, 2006, accessible at: http://www.ofcom.org.uk/consult/condocs/sur/

[10] S. Thajchayapong and J. M. Peha, "Mobility Patterns in Microcellular Wireless Networks," IEEE Transactions on Mobile Computing, Vol. 5, No. 1, January 2006, pp. 52-63

[11] J. Laiho-Steffens, A. Wacker and P. Aikio, "The impact of the radio network planning and site configuration on the WCDMA network capacity and quality of service," Proc. IEEE VTC, vol. 2, pp. 10061010, Tokyo, Japan, 2000. 